\title{
Mammography behavior after receiving a negative BRCA1 mutation test result in the Ashkenazim: A community-based study
}

\author{
Sharon E. Plon, $M D, P h D, F A C M G^{1,2}$, Leif E. Peterson, $P h D^{3}$, Lois C. Friedman, $P h D^{4}$, and \\ C. Sue Richards, PhD, FACMG ${ }^{2}$
}

\begin{abstract}
Purpose: To define the impact of a negative BRCA1 test result on subsequent breast cancer screening behavior in women. Methods: Longitudinal study of a community-based sample of Ashkenazi Jews offered testing for the 185delAG BRCA1 mutation in 1996. Of 309 participants, 118 women were mutation negative, of average risk (based on family history of cancer), unaffected with breast cancer, and provided complete data at baseline, and Year 1 and Year 2 follow-up questionnaires. Results: Women age 50 and older had $91.7 \%$ compliance with mammography for the year prior to entry (baseline), 88.3\% during Year 1, 91.7\% during Year 2 (no significant change; $P=0.775)$. Women under age 50 demonstrated an increase in mammography $(49.2 \%$ at baseline, $62.7 \%$ Year 1, and 67.1\% Year 2; $P=0.035)$. Both groups demonstrated significant decreases in breast cancer worry and perceived risk. Logistic regression analysis on having a mammogram at Year 2 showed that age, physician recommendation, worry, and perceived risk were all significant. Conclusion: Receipt of negative BRCA1 test results in a cohort of Ashkenazi Jewish women did not have a negative impact on mammography behavior 2 years after genetic testing. Genetics in Medicine, 2000:2(6):307-311.
\end{abstract}

Key Words: BRCA1 mammography, breast cancer, screening behavior, genetic testing

Approximately one in eight women in the United States eventually will develop breast cancer. ${ }^{1}$ This risk is higher for women of Ashkenazi Jewish ancestry. ${ }^{2}$ Initial studies of mutations in the $B R C A 1$ breast cancer susceptibility gene revealed a deleterious founder mutation (185delAG) with a carrier frequency of $1 \%$ in the Ashkenazi Jewish population. ${ }^{3,4}$ This germline mutation is associated with a higher rate and an earlier onset of both breast and ovarian cancer. ${ }^{5.6}$ Another $B R C A 1$ mutation (5382insC) and a BRCA2 mutation (6174delT) were discovered to be frequent in the Ashkenazi population subsequent to the initiation of this study. ${ }^{7,8}$ Overall, approximately 1 in 40 Ashkenazi Jews carries one of these three cancer susceptibility mutations. ${ }^{9,10}$

The identification of breast cancer susceptibility genes provides the opportunity for DNA testing of at-risk individuals. It is important to study the impact of a negative test result on cancer screening behaviors because ( 1 ) the majority of individuals undergoing testing will receive negative test results (in one study, only $18 \%$ of a high-risk population tested positive for a mutation in $B R C A 1$ or $B R C A 2^{11}$ ) and (2) women with negative $B R C A 1$ and $B R C A 2$ test results retain a substantial lifetime risk

From the Departments of 'Petiatrics, Molecular and Human Gicnetics, 'Medicimt, ant 'Psychiatry and Beharioral Science, Baylor College' of Medicime, Heuston. Texals.

Dr. Sharon F. Plon, Tixas Children's Hospital MC 3-3320, 6621 Fimmin Street. Houston. $7 \mathrm{X}$ 77030 .

Received: Séptember 7, 2000.

Accepted: Septemher 28, 2000 of developing breast cancer. It is important to determine whether individuals who receive negative $B R C A 1$ or $B R C A 2$ test results continue to follow cancer surveillance guidelines and remain aware that they are still at risk of developing breast cancer. Studying the impact of DNA testing on Ashkenazim is especially relevant, because the frequency of these founder mutations and the lower cost of DNA testing for founder mutations facilitates testing in this population.

The purpose of this study was to examine longitudinally the psychological and medical impact of DNA testing for a common mutation, BRCA1 185delAG, in a community-based sample of Ashkenazi Jews. For this analysis, we studied only those participants who received negative test results and did not have significant family histories of cancer. We hypothesized that negative test results would be associated with a decrease in mammography behavior over time. In addition, we examined the impact of other factors on mammography behavior, including breast cancer risk perception, breast cancer worry, and physician recommendation.

\section{METHODS}

\section{Procedures}

A full description of the initial testing portion of this study can be found in Richards et al.' 2 The Baylor College of Medicine Institutional Review Board approved the study and informed consent for DNA testing and longitudinal follow-up was obtained after the initial educational session. The study 
was advertised in the Houston community, including local publications and newsletters of Jewish organizations. Eligibility included all adult men and women of at least 50\% Ashkenazi ancestry independent of personal or family history of cancer. People interested in participating were invited to attend 90-minute educational/enrollment sessions held at either Baylor College of Medicine or the Jewish Community Center of Houston. The sessions included a 45-minute slide presentation. The first half covered a number of topics, including a summary of risk factors for breast cancer, breast and ovarian cancer genetics, cancer risk in $B R C A 1$ mutation carriers, and the discovery of the $185 \mathrm{del} A G$ mutation in the BRCAl gene. The meaning of both a positive and negative mutation test were reviewed as well as recommendations to continue cancer screening guidelines (for breast and colon cancer) for individuals receiving a negative test result. The second half described the goals of the study and reviewed the protocol and informed consent documents. At the end of the session participants who elected to join the study were asked to fill out the questionnaires described below and were offered testing (with disclosure of results) for the $185 \mathrm{del} A \mathrm{G}$ mutation.

Information with regard to mammography behavior, personal and family cancer histories, doctor recommendation, breast cancer risk perception, and breast cancer worry were obtained by self-administered questionnaires. Questionnaires were filled out at entry prior to testing (baseline) and by mail at 1 month, 6 months, 1 year, and 2 years after receipt of test results. Testing with disclosure of results was performed in the Spring of 1996 only for the 185 delAG mutation. Negative DNA test results were disclosed by letter, and these participants were advised to follow guidelines for breast cancer screening, which at that time included annual mammograms for women age 50 and older.

\section{Study population}

Overall, 12 enrollment sessions were held, resulting in 309 subjects (93\% of attendees) electing to participate in the study. As described in detail in Richards et al., ${ }^{12}$ this was a well-educated cohort with $45 \%$ having college education and $50 \%$ some graduate study. For the study reported here, we eliminated from this analysis 37 males, 22 subjects who declined testing or had positive test results for $185 \mathrm{del} A \mathrm{G}, 32$ females who reported personal histories of breast cancer at baseline, and 3 who failed to report their year of birth. This left us with 215 participants. Of this group, 203 were defined as average risk based on family history and 12 were defined as increased risk as described below. One hundred ninety-three average-risk women provided responses for mammography behavior, breast cancer worry, and perceived risk at baseline.

\section{Measures}

The self-report measures used in the present study at baseline and for each of the follow-up questionnaires included demographic information (age and education), personal and family cancer histories, mammography behavior, physician recommendation of mammography, breast cancer risk perception, and breast cancer worry.

\section{Cancer history}

Participants indicated whether they ever had been diagnosed with breast, ovarian, colon or "other" cancers. To assess family cancer history, participants indicated first- and seconddegree relatives who had been diagnosed with breast, ovarian, and/or colon cancer and age at diagnosis. A positive family history for this study was defined as having one first-degree or two second-degree relatives with breast cancer (at least one under age 50 ) or ovarian cancer. Participants were defined as "average" risk if they did not meet these criteria based on family history.

\section{Mammography behavior}

Participants were asked in each follow-up questionnaire if they had a mammogram during the past year (for baseline measure) or since they last filled out the questionnaire (for follow-up questionnaires). Year 1 mammography behavior was considered positive if they answered "yes" to this question at either the 1 month, 6 months, or 1 year follow-up questionnaire.

\section{Physician recommendation of mammography}

Participants were asked the following yes/no question at baseline, Year 1, and Year 2, "In the past 12 months, did your doctor recommend that you get the following procedures?" with mammography being the first procedure listed.

\section{Breast cancer worry}

Participants were asked, "How much do you worry that you will get breast cancer some day?" with the choices being 1-not at all, 2-a little, 3-somewhat, and 4-a lot.

\section{Breast cancer perceived risk}

Participants were asked "What do you think your chances are of getting breast cancer some day?" with the same choices as noted for worry.

\section{Statistical analysis}

Repeated measures analysis of variance (RMANOVA, SPSS version 9.0) was conducted to test for no significant change in mammography, breast cancer-specific worry, and perceived risk scores over the 3-year period (baseline and 2 years of follow-up). ${ }^{13}$ Only records with complete information for the three follow-up periods were used. Logistic regression analysis was conducted to assess the effects of age category (age 50+ vs. age $<50$ ), physician recommendation (yes vs. no), and oneunit increases in breast cancer worry and perceived risk scores on the Year 2 questionnaires on the odds of having mammography during the last (second) follow-up period in the study.

\section{Attrition analysis}

One hundred ninety-three women provided responses for mammography behavior, breast cancer worry, and perceived risk at baseline. Complete information on these three measures was provided at baseline, Year 1 , and Year 2 questionnaires by 
118 women. Comparison between these two groups for measures of baseline mammography, perceived risk, and worry about breast cancer is shown in Table 1. Unpaired $t$ tests (STATA version 5.0) showed no significant differences between the two groups ( $P$ values 0.5$)$ for the three measures.

\section{RESULTS}

At the time the average-risk participants received their negative test result, they were informed that recommendations for breast cancer surveillance included annual mammography beginning at age 50 . Therefore, in this study, we specifically examined mammography behavior of women age 50 and older separately from that of younger women.

Participants were 118 females whose mean age was 51 years ( 58 below age 50 , and 60 age 50 and older) with a range from 26 to 75 . These women all provided complete data for mammography, worry, and perceived breast cancer risk at baseline, Year 1 , and Year 2. RMANOVA results for these three measures are shown in Table 2. For the older cohort, $91.7 \%$ reported having a mammogram in the year prior to entry and $88.3 \%$ during Year 1, and $91.7 \%$ during Year 2. These differences are not statistically significant. Analysis of the non-Hispanic Caucasian United States population in 1998 shows $69.8 \%$ had a mammogram in the past 2 years. ${ }^{14}$ Thus, for this age group, receipt of a negative $B R C A l$ result did not alter their very high level of compliance with mammography recommendations. This is despite finding that their worry of developing breast cancer (2.68 baseline, 2.30 Year 1, and 2.45 Year 2; $P=0.002$ ) and perceived risk ( 2.68 baseline, 2.65 Year 1, and 2.58 Year 2; $P=0.027)$ declined significantly over the 2 years of follow-up. We performed a similar analysis, including all women $(N=$ 71) for whom we have complete mammography data (but may be missing some worry or risk data), which also reveals a nonsignificant change $(P=0.469)$.

Soon after women in our study received their test results (July of 1996), there was substantial re-evaluation of the benefit for routine mammography in women under age 50 . The National Cancer Institute convened a consensus panel ${ }^{15}$ and in April of 1997 issued new guidelines publicized in many journals including recommendation of mammography every 1 to 2 years for women between ages of 40 and 50. ${ }^{16,17}$ Although we did not notify the cohort about these changes, women may have read about them in the lay literature or received new recommendations for mammography from their personal physicians. Women in our cohort under age 50 significantly increased their mammography behavior from $46.6 \%$ at baseline to $56.9 \%$ during Year 1 and $65.5 \%$ during Year $2(P<$ 0.035 ). These women also demonstrated a significant decline in perceived risk and worry about breast cancer over the 2 years of follow-up. Analogously, 67 women below age 50 with complete mammography data but potentially missing worry and perceived risk data also had a significant increase in mammography from baseline until the end of follow-up year 2 ( $P=$ $0.025)$.

Of the group below age $50(N=58)$ for which we have complete information, 44 women were between the ages of 40 and 49. Analysis of this subset also showed increases in mammography behavior from $59 \%$ at baseline to $68 \%$ during Year 1 and $77 \%$ during Year 2. Comparison with the non-Hispanic Caucasian United States population from ages 40 to 49 in 1998 demonstrates that $64 \%$ had a mammogram in the past 2 years.

Logistic regression analysis with the dependent variable based on having mammography during Year $2(N=144)$ demonstrated that age, physician recommendation, worry about developing breast cancer, and perceived risk of developing breast cancer as reported on the Year 2 questionnaires were all statistically significant predictors of mammography (Table 3). Interestingly, the odds ratios for reporting mammography in Year 2 were $6.9[95 \%$ confidence interval (CI), 2.47-19.27] for women over age 50 (vs. $<50), 6.2(95 \% \mathrm{CI}, 2.35-16.36)$ for women with a physician recommendation (vs. no recommendation), and 3.76 (95\% CI, 1.410.12) for a one-unit increase on the perceived risk scale but only 0.35 (95\% CI, 0.16-0.79) for a one-unit increase on the breast cancer worry scale. There was no significant effect modification (interaction) between any of these factors.

\section{DISCUSSION}

In our analysis of longitudinal data from a cohort of average risk Ashkenazi women receiving negative results for $B R C A 1$ testing, there was no decline in mammography behavior after receiving the test result. This cohort started out with excellent compliance of $89 \%$ for women age 50 and older and continued to have excellent compliance 2 years after receiving test results. The high level at baseline may reflect a bias in women joining the study. Potential biases include that women who volunteered to participate in the study may have higher compliance

Table 1

Attrition analysis of the cohort

\begin{tabular}{lccc}
\hline Scale & Whole group $(n=193)$ & Complete data $(n=118)$ & $P$ value \\
\hline Having mammography $(\%)$ & $68.0(0.03)$ & $69.5(0.04)$ & 0.76 \\
Worry & $2.74(0.06)$ & $2.66(0.08)$ & 0.44 \\
Perceived risk & $2.9(0.05)$ & $2.86(0.07)$ & 0.60 \\
\hline
\end{tabular}

Mean values (standard error) for all average risk, test negative women at baseline versus women with complete data for baseline, Year 1 and Year 2 questionnaires.

"Unpaired $t$ test with null hypothesis $\mathrm{H}_{0}: \mu_{1}-\mu_{2}=0$. 
Table 2

Mean values (standard error) of proportion having mammography (\%) and scores for worry and perceived risk

\begin{tabular}{lcccc}
\hline & \multicolumn{5}{c}{ Follow-up period } \\
\cline { 2 - 4 } & Baseline & Year 1 & Year 2 & P value \\
\hline Women age $\geq 50(n=60)$ & $91.7(3.6)$ & $88.3(4.2)$ & $91.7(3.6)$ & 0.755 \\
Having mammography $(\%)$ & $2.68(0.12)$ & $2.30(0.11)$ & $2.45(0.11)$ & 0.002 \\
Worry & $2.82(0.10)$ & $2.65(0.09)$ & $2.58(0.08)$ & 0.027 \\
Perceived risk & & & & \\
Women age $<50(n=58)$ & $46.6(6.6)$ & $56.9(6.6)$ & $65.5(63)$ & 0.035 \\
Having mammography $(\%)$ & $2.64(0.12)$ & $2.33(0.10)$ & $2.36(0.09)$ & 0.003 \\
Worry & $2.90(0.09)$ & $2.67(0.09)$ & $2.69(0.08)$ & 0.012 \\
Perceived risk & & & & \\
\hline
\end{tabular}

Hypothesis tests for no change in value over the 3 -year period were conducted with repeated-measures analysis of variance and were based on the Huynh-Feldt ${ }^{2-2}$ test.

Table 3

Logistic regression for having mammography during Year 2 for women of all ages $(n=144)$

\begin{tabular}{lcccc}
\hline Response on Year 2 questionnaire & OR & SE (OR) & $P$ value & $95 \%$ CI \\
\hline Age 50+ (vs. age $<50)$ & 6.90 & 3.62 & $<0.001$ & $2.47-19.27$ \\
Doctor recommendation & 6.20 & 3.07 & $<0.001$ & $2.35-16.36$ \\
Cancer specific worry & 0.35 & 0.15 & 0.012 & $0.16-0.79$ \\
Perceived cancer risk & 3.76 & 1.90 & 0.009 & $1.4-10.12$ \\
\hline
\end{tabular}

Odds ratio (OR), standard error (SE), and $95 \%$ confidence intervals (CI). Reference category (i.e., odds ratios $=1$ ) based on women age $<50$ without doctor recommendation. Odds ratios for cancer-specific worry and perceived risk based on one-unit increase in worry and perceived cancer risk scales.

than the general Ashkenazi population. There does not appear to be selective attrition, as the women for whom we have complete data had similar mammography behavior and breast cancer worry and risk perception at baseline as the overall cohort. Therefore, the concern that a negative test result would result in decline in cancer surveillance methods does not appear justified with regard to mammography screening for breast cancer. In particular, younger women (age $<50$ ) in this cohort had a statistically significant increase in mammography. This finding may be a direct impact of participating in our study, learning about the possibility of early-onset breast cancer, and receiving follow-up questionnaires, or it potentially may be a response to changes in nationally publicized recommendations by the National Cancer Institute for increased mammography behavior in women under age 50 .

Although a number of investigators have noted that moderate levels of cancer-specific worry appear to be positively correlated with mammography behavior, ${ }^{18}$ our cohort did not demonstrate any decline in mammograms even though cancer worry did decline from baseline. This may be because the decline in worry still resulted in the mean score of the cohort in the mildly to moderately worried range. In contrast, logistic regression demonstrated that worry did have a negative impact on mammography behavior. This finding is consistent with the work of Lerman and colleagues ${ }^{19,20}$ who demonstrated that high levels of cancer-specific worry can intrude on surveillance behaviors. Thus, women seen either in the context of genetic counseling or primary care visits who demonstrate high levels of cancer worry may require additional education and counseling to ensure appropriate cancer screening and surveillance.

Overall, this cohort of well-educated Ashkenazi women who received negative $B R C A 1$ test results still complied with their physicians' recommendations and continued to correctly perceive that they remained at risk of developing breast cancer, necessitating mammography surveillance. One challenge to geneticists and oncologists is ensuring that women who may be less medically sophisticated than our cohort understand these issues as well. For example, in one study, genetic counseling about breast cancer risk resulted in a decrease in mammography behavior for less-educated participants. ${ }^{21}$ The need for continued surveillance in women who test negative for breast cancer susceptibility gene mutations is highlighted by the diagnosis of breast cancer in six "average" risk women in the Ashkenazi cohort during the 2 follow-up years of this study.

\section{Acknowledgments}

This study was supported in part by the Jewish Institute of Medical Research Endowment Fund and the JIMR/Glesby Endowment Fund of the Endowment Fund of the Jewish Community of Houston and by grants K07-CA78199-02 (to LEP) 
and U24-CA78142-02 (to SEP) from the National Cancer Institute.

\section{References}

1. American Cancer Society. Cancer facts and figures-1997. Atlanta: American Cancer Society, 1997.

2. Claus EB, Risch N, Thompson WD. Genetic analysis of breast cancer in the cancer and steroid hormone study. Am J Hum Genet 1991;48:232-242.

3. Goldgar DE, Reilly PR. A common BRCA1 mutation in the Ashkenazim. Nat Genet 1995;11:113-114

4. Struewing JP, Abeliovich D, Peretz $\mathrm{T}$, et al. The carrier frequency of the $B R C A$ 185 delAG mutation is approximately 1 percent in Ashkenazi lewish individuals. Nat Genet 1995;11:198-200.

5. Struewing JP, Hartge $P$, Wacholder $S$, et al. The risk of cancer associated with specific mutations of BRCA1 and BRCA2 among Ashkenazi Jews. N Engl J Med 1997;336: 1401-1408.

6. Levy-Lahad E, Catane R, Eisenberg S, et al. Founder BRCA1 and BRCA2 mutations in Ashkenazi Jews in Israel: frequency and differential penetrance in ovarian cancer and in breast-ovarian cancer families. Am J Hum Genet 1997:60:1059-1067.

7. Neuhausen S, Gilewski T, Norton L, et al. Recurrent BRCA2 6174delT mutations in Ashkenazi Jewish women affected by breast cancer. Nat Genet 1996;13:126-128.

8. Couch FJ, Farid LM, DeShano ML, et al. BRCA2 germline mutations in male breast cancer cases and breast cancer families. Nat Genet 1996;13:123-125.

9. Roa BB, Boyd AA, Volcik K, Richards CS. Ashkenazi Jewish population frequencies for common mutations in BRCA1 and. BRCA2. Nat Genet 1996;14:185-187.

10. Oddoux C, Struewing JP, Clayton $\mathrm{CM}$, et al. The carrier frequency of the BRCA2 6174 delT mutation among Ashkenazi Jewish individuals is approximately $1 \%$. Nat Genet 1996;14:188-190.
11. Shattuck-Eidens D, Oliphant A, McClure M, et al. BRCA1 sequence analysis in women at high risk for susceptibility mutations: risk factor analysis and implications for genetic testing. JAMA 1997;278:1242-1250.

12. Richards CS, Ward PA, Roa BB, et al. Screening for 185delAG in the Ashkenazim Am J Hum Genet 1997;60:1085-1098.

13. Cole JWL, Grizzle JE. Applications of multivariate analysis of variance to repeated measurements experiments. Biometrics 1966;22:810-828.

14. National Center for Health Statistics and Centers for Disease Control. Health, United States, 2000. Washington, DC: US Government Printing Office, 2000.

15. Breast Cancer Screening for Women Ages 40-49. NIH Consensus Statement, 1997.

16. Eastman $\mathrm{P} . \mathrm{NCl}$ adopts new mammography screening guidelines for women [news]. I Natl Cancer Inst 1997;89:538-539.

17. Taubes G. NCI reverses one expert panel, sides with another [news]. Science 1997; 276:27-28.

18. Diefenbach MA, Miller SM, Daly MB. Specific worry about breast cancer predicts mammography use in women at risk for breast and ovarian cancer. Health Psychol 1999; 18:532-536.

19. Lerman C, Rimer B, Trock B, Balshem A, Engstrom PF. Factors associated with repeat adherence to breast cancer screening. Prev Med 1990;19:279-290.

20. Lerman C, Daly M, Sands C, et al. Mammography adherence and psychologica distress among women at risk for breast cancer. J Natl Cancer Inst 1993;85:10741080.

21. Schwartz MD, Rimer BK, Daly M, Sands C, Lerman C. A randomized trial of breast cancer risk counseling: the impact on self- reported mammography use. Am J Public Health 1999;89:924-926.

22. Huynh H, Feldt LS. Conditions under which mean square ratios in repeated measurements designs have exact F-distributions. J Am Stat Assoc 1970;65:1582 1589. 